Supplement of Clim. Past, 12, 129-136, 2016

http://www.clim-past.net/12/129/2016/

doi:10.5194/cp-12-129-2016-supplement

(C) Author(s) 2016. CC Attribution 3.0 License.

(c) (i)

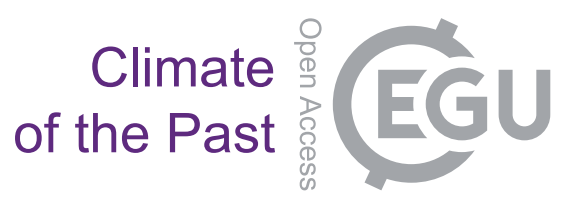

Supplement of

\title{
Climate-driven expansion of blanket bogs in Britain during the Holocene
}

A. V. Gallego-Sala et al.

Correspondence to: A. V. Gallego-Sala (a.gallego-sala@exeter.ac.uk)

The copyright of individual parts of the supplement might differ from the CC-BY 3.0 licence. 
This Supplementary file contains information on the individual sites used for the basal ages compilation, including latitude and longitude $\left(\right.$ decimal $\left.{ }^{\circ}\right)$, altitude $(\mathrm{m})$, source reference, lowermost radiocarbon date $(14 \mathrm{C}$ date) and its uncertainty (error) in years $\mathrm{BP}$, the age of this date in calibrated years BP (and uncertainty), the depth of the peat at the site, the dating method used to establish the basal age of the peat (either a radiocarbon-based chronology, $14 \mathrm{C}$, or a pollen-based chronology) and the region to which the site is assigned in our analyses.

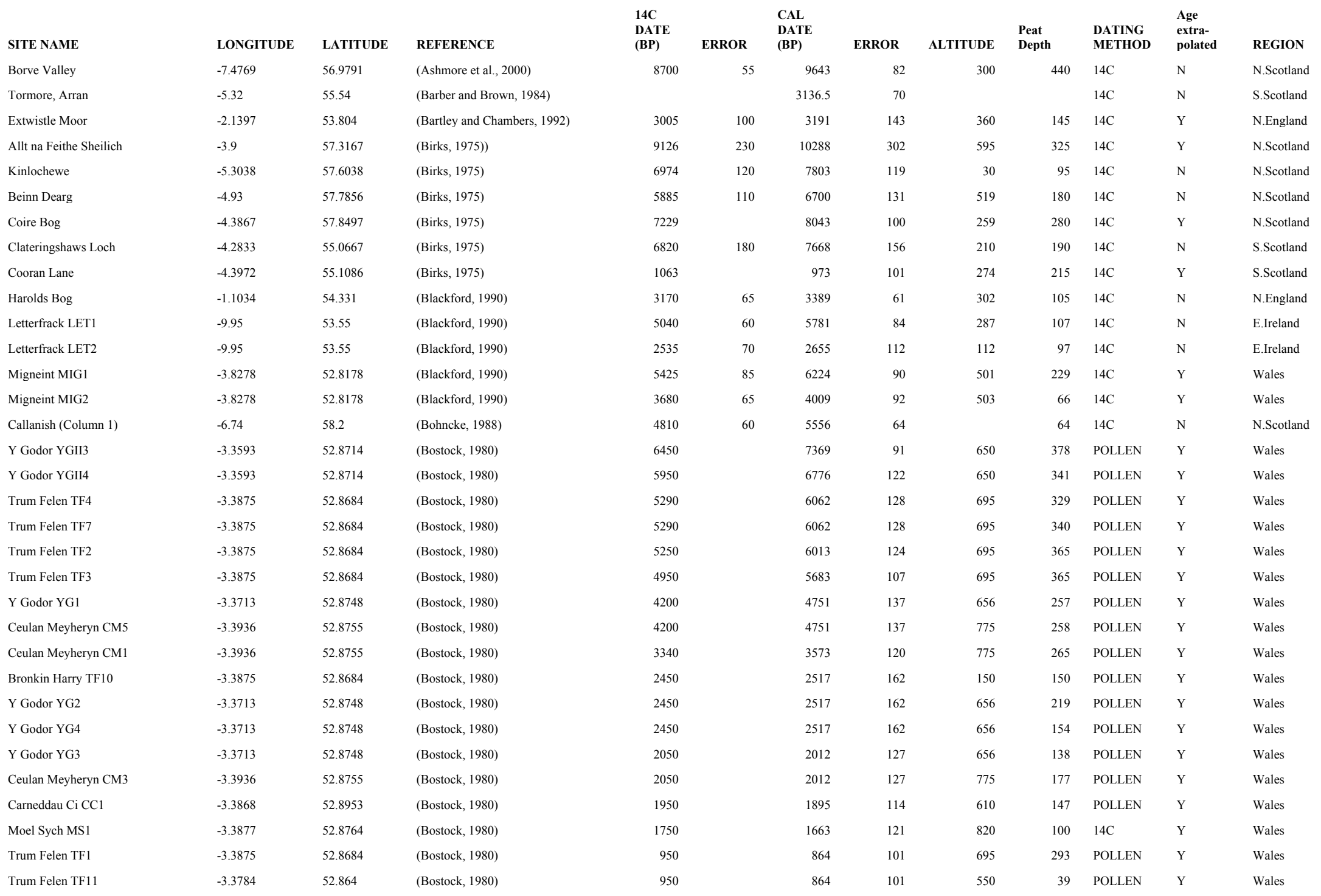




\begin{tabular}{|c|c|c|c|}
\hline SITE NAME & LONGITUDE & LATITUDE & REFERENCE \\
\hline Coire Seilich & -4.6994 & 56.5811 & (Bridge et al., 1990) \\
\hline Clashgour C Rannoch Moor & -4.8516 & 56.548 & (Bridge et al., 1990) \\
\hline Gleann Fuar Rannoch Moor & -4.8496 & 56.5211 & (Bridge et al., 1990) \\
\hline Clashgour A Rannoch Moor & -4.8332 & 56.5412 & (Bridge et al., 1990) \\
\hline Caislean, Achill Island & -10.133 & 53.9833 & (Caseldine et al., 2005) \\
\hline Caislean, Achill Island & -10.133 & 53.9833 & (Caseldine et al., 2005) \\
\hline Brecon Beacons CAR 51 & -3.5 & 51.8667 & (Chambers, 1981, 1982) \\
\hline Cefn Fford CAR 9 CFF & -3.585 & 51.7168 & (Chambers, 1981, 1982) \\
\hline Coed Taf A & -3.4686 & 51.7867 & (Chambers, 1983) \\
\hline Coed Taf B & -3.4686 & 51.7867 & (Chambers, 1983) \\
\hline Cefn Gwernffrwd CG & -3.8494 & 52.1284 & (Chambers, 1981) \\
\hline Coed Taf C & -3.4686 & 51.7867 & (Chambers, 1983) \\
\hline Talla Moss & -3.3429 & 55.4692 & (Chambers et al., 1997) \\
\hline Coom Rigg B & -2.4874 & 55.1044 & (Chapman, 1964) \\
\hline Cross Lochs Fen A (1) & -3.9122 & 58.3872 & (Charman, 1994, 1990) \\
\hline Cross Lochs Fen A (5) & -3.9156 & 58.3872 & (Charman, 1994, 1990) \\
\hline Cross Lochs Fen A (7) & -3.9173 & 58.3871 & (Charman, 1994, 1990) \\
\hline Cross Lochs Fen A (3) & -3.9139 & 58.3871 & (Charman, 1994, 1990) \\
\hline Cross Lochs Fen A (2) & -3.9122 & 58.3872 & (Charman, 1994, 1990) \\
\hline Cross Lochs Fen A (4) & -3.9139 & 58.3872 & (Charman, 1994, 1990) \\
\hline Cross Lochs Fen A (6) & -3.9156 & 58.3872 & (Charman, 1994, 1990) \\
\hline Cross Lochs 1 & -3.9345 & 58.3878 & (Charman, 1992) \\
\hline Cross Lochs 4 & -3.9345 & 58.3878 & (Charman, 1992) \\
\hline Cross Lochs 3 & -3.9345 & 58.3878 & (Charman, 1992) \\
\hline Cross Lochs 2 & -3.9345 & 58.3878 & (Charman, 1992) \\
\hline St Helena B & -0.9 & 54.4 & (Cundill, 1977) \\
\hline Bluewath Beck & -0.8595 & 54.3949 & (Cundill, 1977) \\
\hline Collier Gill Head & -0.9 & 54.4 & (Cundill, 1977) \\
\hline White Gill & -0.9 & 54.4 & (Cundill, 1977) \\
\hline Trough House & -0.9233 & 54.3997 & (Cundill, 1977) \\
\hline Loose House & -0.9 & 54.4 & (Cundill, 1977) \\
\hline Glaisdale Moor & -0.8925 & 54.3994 & (Cundill, 1977) \\
\hline Blakey Slip & -0.9 & 54.4 & (Cundill, 1977) \\
\hline Blacky Landslip & -0.9 & 54.4 & (Cundill, 1977) \\
\hline Pike Hill Moss & -0.8309 & 54.3988 & (Cundill, 1977) \\
\hline Howdale Hill & -1.0157 & 54.4005 & (Cundill, 1977) \\
\hline Yarsley Moss & -0.8465 & 54.39 & (Cundill, 1977) \\
\hline St Helena A & -0.9 & 54.4 & (Cundill, 1977) \\
\hline
\end{tabular}

\begin{tabular}{|c|c|c|c|}
\hline $\begin{array}{l}\text { 14C } \\
\text { DATE } \\
\text { (BP) }\end{array}$ & ERROR & $\begin{array}{r}\text { CAL } \\
\text { DATE } \\
\text { (BP) }\end{array}$ & ERROR \\
\hline 9779 & 90 & 11197 & 85 \\
\hline 9370 & 60 & 10588 & 76 \\
\hline 8310 & 50 & 9327 & 82 \\
\hline 6460 & 50 & 7386 & 48 \\
\hline 9850 & 300 & 11310 & 470 \\
\hline 8860 & 30 & 9981 & 101 \\
\hline 4380 & 70 & 4953 & 114 \\
\hline 3625 & 80 & 3937 & 108 \\
\hline 1435 & 55 & 1329 & 39 \\
\hline 1310 & 70 & 1243 & 66 \\
\hline 3465 & 70 & 3727 & 94 \\
\hline 6885 & 110 & 7717 & 103 \\
\hline 6275 & & 7193 & 118 \\
\hline 4000 & 0 & 4468 & 143 \\
\hline 9400 & 50 & 10624 & 65 \\
\hline 7200 & & 8016 & 96 \\
\hline 5650 & & 6431 & 106 \\
\hline 5250 & & 6013 & 124 \\
\hline 4900 & & 5633 & 100 \\
\hline 3850 & & 4258 & 149 \\
\hline 3550 & & 3839 & 133 \\
\hline 9170 & 90 & 10322 & 111 \\
\hline 8835 & 165 & 9903 & 259 \\
\hline 6805 & 50 & 7642 & 38 \\
\hline 4035 & 55 & 4504 & 82 \\
\hline 5500 & 1000 & 6251 & 1129 \\
\hline 5500 & 1000 & 6251 & 1129 \\
\hline 5500 & 1000 & 6251 & 1129 \\
\hline 5500 & 1000 & 6251 & 1129 \\
\hline 5500 & 1000 & 6251 & 1129 \\
\hline 5500 & 1000 & 6251 & 1129 \\
\hline 5500 & 1000 & 6251 & 1129 \\
\hline 5500 & 1000 & 6251 & 1129 \\
\hline 5500 & 1000 & 6251 & 1129 \\
\hline 2500 & 500 & 2570 & 603 \\
\hline 2500 & 500 & 2570 & 603 \\
\hline 2500 & 500 & 2570 & 603 \\
\hline 2500 & 500 & 2570 & 603 \\
\hline
\end{tabular}

\begin{tabular}{|c|c|c|c|c|}
\hline JDE & $\begin{array}{r}\text { Peat } \\
\text { Depth }\end{array}$ & $\begin{array}{l}\text { DATING } \\
\text { METHOD }\end{array}$ & $\begin{array}{l}\text { Age } \\
\text { extra- } \\
\text { polated }\end{array}$ & REGION \\
\hline 300 & 433 & $14 \mathrm{C}$ & $\mathrm{Y}$ & S.Scotland \\
\hline 293 & 333 & $14 \mathrm{C}$ & $\mathrm{N}$ & S.Scotland \\
\hline 200 & 230 & $14 \mathrm{C}$ & $\mathrm{N}$ & S.Scotland \\
\hline 180 & 174 & $14 \mathrm{C}$ & $\mathrm{N}$ & S.Scotland \\
\hline 50 & 291 & $14 \mathrm{C}$ & $\mathrm{N}$ & W.Ireland \\
\hline 50 & 233 & $14 \mathrm{C}$ & $\mathrm{N}$ & W.Ireland \\
\hline 715 & 102 & $14 \mathrm{C}$ & $\mathrm{N}$ & Wales \\
\hline 600 & 25 & $14 \mathrm{C}$ & $\mathrm{N}$ & Wales \\
\hline 400 & 29 & $14 \mathrm{C}$ & $\mathrm{N}$ & Wales \\
\hline 400 & 71 & $14 \mathrm{C}$ & $\mathrm{N}$ & Wales \\
\hline 400 & 69 & $14 \mathrm{C}$ & $\mathrm{N}$ & Wales \\
\hline 400 & 354 & $14 \mathrm{C}$ & $\mathrm{Y}$ & Wales \\
\hline 600 & 120 & $14 \mathrm{C}$ & Y & S.Scotland \\
\hline 300 & 150 & POLLEN & Y & N.England \\
\hline 158 & 367 & $14 \mathrm{C}$ & Y & N.Scotland \\
\hline 162 & 215 & POLLEN & $\mathrm{N}$ & N.Scotland \\
\hline 164 & 184 & POLLEN & $\mathrm{N}$ & N.Scotland \\
\hline 160 & 168 & POLLEN & $\mathrm{N}$ & N.Scotland \\
\hline 159 & 160 & POLLEN & $\mathrm{N}$ & N.Scotland \\
\hline 161 & 156 & POLLEN & $\mathrm{N}$ & N.Scotland \\
\hline 163 & 110 & POLLEN & $\mathrm{N}$ & N.Scotland \\
\hline 148 & 138 & $14 \mathrm{C}$ & $\mathrm{N}$ & N.Scotland \\
\hline 118 & 172 & $14 \mathrm{C}$ & $\mathrm{N}$ & N.Scotland \\
\hline 168 & 207 & $14 \mathrm{C}$ & $\mathrm{N}$ & N.Scotland \\
\hline 168 & 52 & $14 \mathrm{C}$ & $\mathrm{N}$ & N.Scotland \\
\hline \multirow{13}{*}{365} & \multirow{10}{*}{131} & POLLEN & Y & N.England \\
\hline & & POLLEN & Y & N.England \\
\hline & & POLLEN & Y & N.England \\
\hline & & POLLEN & Y & N.England \\
\hline & & POLLEN & $\mathrm{Y}$ & N.England \\
\hline & & POLLEN & Y & N.England \\
\hline & & POLLEN & Y & N.England \\
\hline & & POLLEN & Y & N.England \\
\hline & & POLLEN & Y & N.England \\
\hline & & POLLEN & Y & N.England \\
\hline & 64 & POLLEN & Y & N.England \\
\hline & \multirow{2}{*}{200} & POLLEN & $\mathrm{Y}$ & N.England \\
\hline & & POLLEN & Y & N.England \\
\hline
\end{tabular}




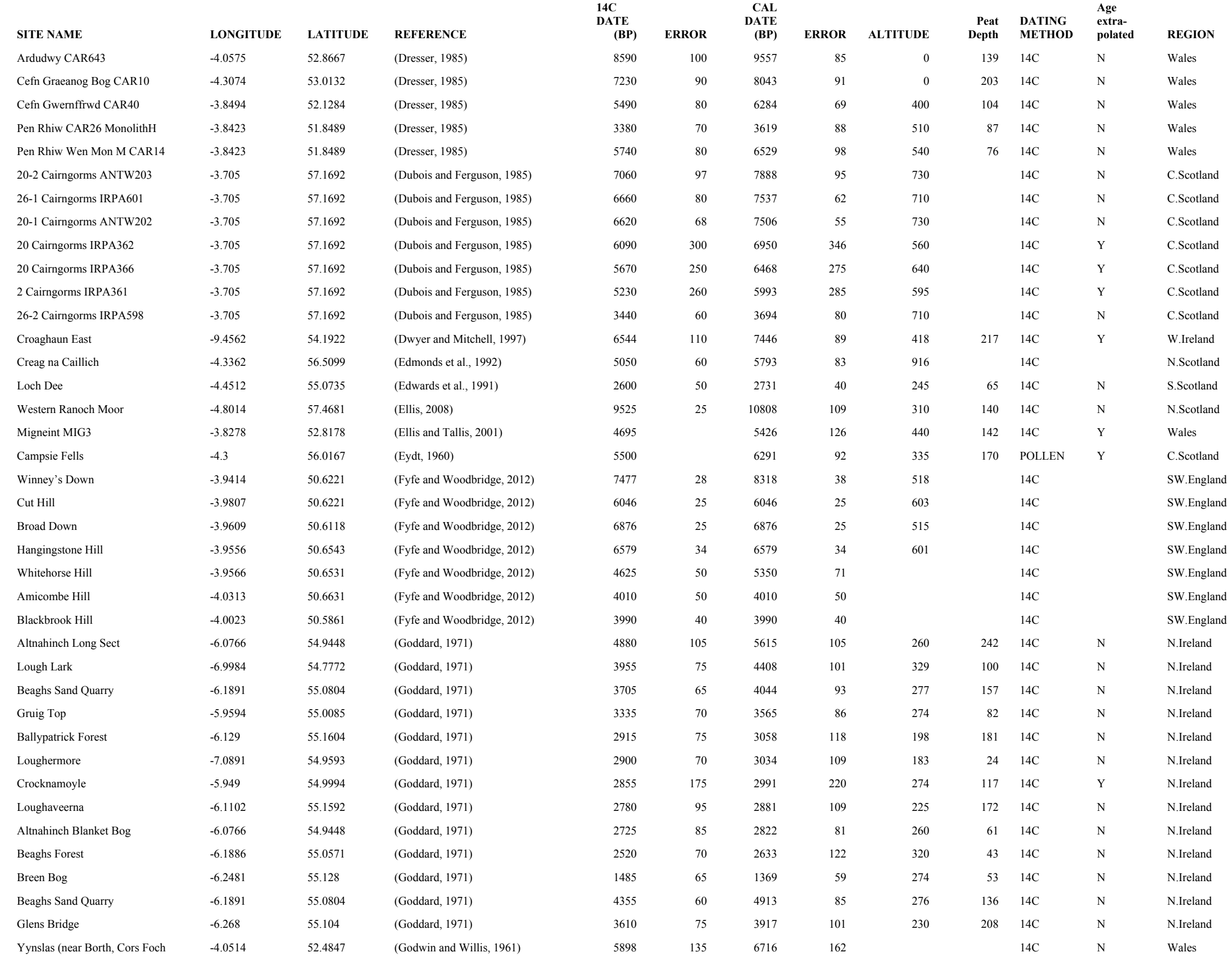




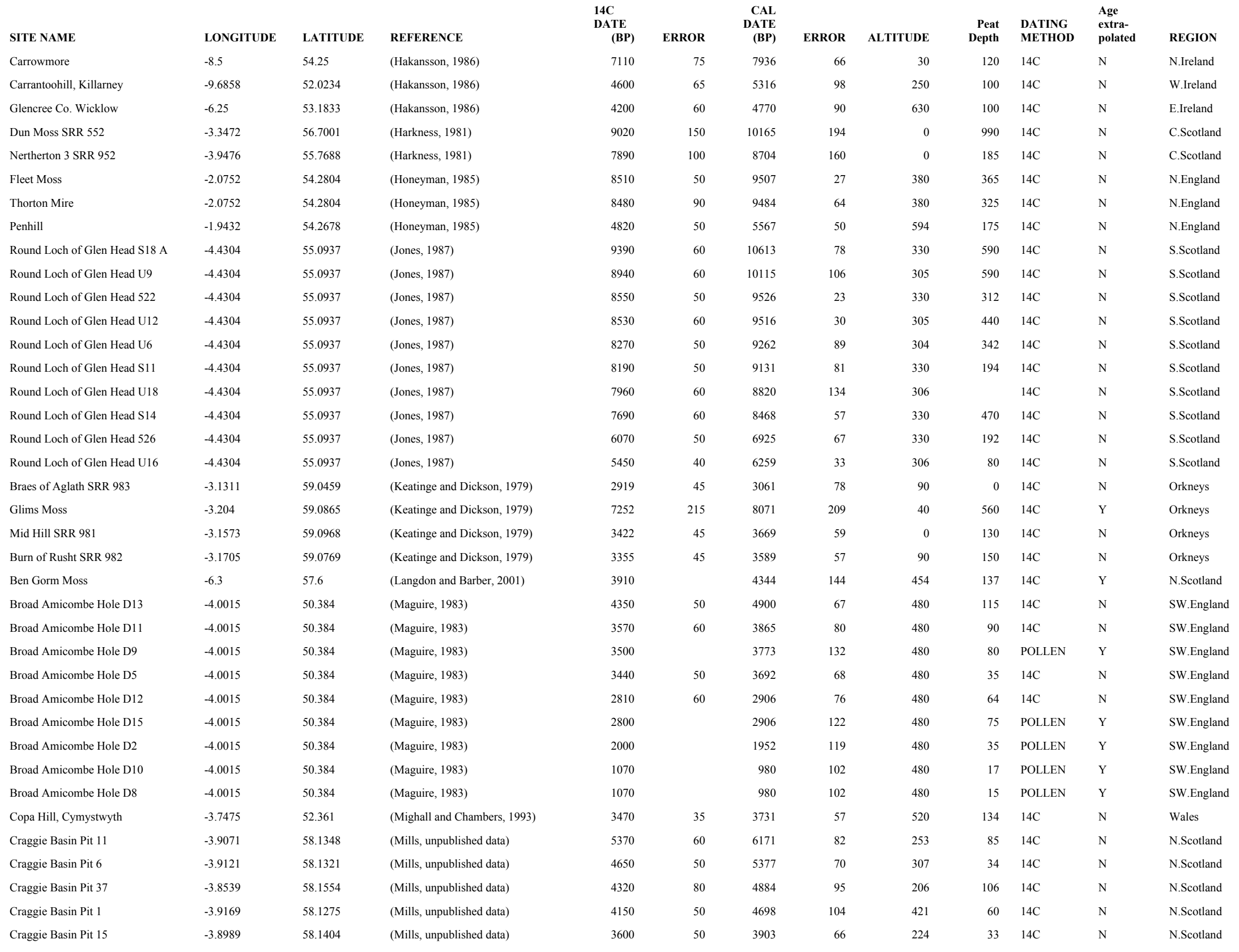




\begin{tabular}{|c|c|c|c|}
\hline SITE NAME & LONGITUDE & LATITUDE & REFERENCE \\
\hline Craggie Basin Pit 19 & -3.8956 & 58.1422 & (Mills, unpublished data) \\
\hline Craggie Basin Pit 23 & -3.8907 & 58.1459 & (Mills, unpublished data) \\
\hline Craggie Basin Pit 34 & -3.8792 & 58.1523 & (Mills, unpublished data) \\
\hline Craggie Basin Pit 29 & -3.8858 & 58.1486 & (Mills, unpublished data) \\
\hline Duartbeg & -5.1309 & 58.2958 & (Moar, 1969) \\
\hline Bellanaboy & -9.59 & 54.3 & $\left(O^{\prime}\right.$ Connell, 1990) \\
\hline Bellanaboy & -9.59 & 54.3 & $\left(O^{\prime}\right.$ Connell, 1990) \\
\hline Glenveagh & -8 & 55.07 & (O'Connell, 1990) \\
\hline Glenveagh & -8 & 55.07 & (O'Connell, 1990) \\
\hline Maughansilly & -9.86 & 51.74 & (O'Connell, 1990) \\
\hline Aukhorn, Caithness M1 & -3.16 & 58.5552 & (Robinson, 1987) \\
\hline Aukhorn, Caithness M3 & -3.16 & 58.5552 & (Robinson, 1987) \\
\hline Bonfield Gill Head & -1.0814 & 54.3542 & (Simmons and Innes, 1981) \\
\hline Waun Felen Fignen B125N & -3.674 & 50.9485 & (Smith and Cloutman, 1988) \\
\hline Waun Felen Fignen EE17E & -3.674 & 50.9485 & (Smith and Cloutman, 1988) \\
\hline Waun Felen Fignen F117S & -3.674 & 50.9485 & (Smith and Cloutman, 1988) \\
\hline Waun Felen Fignen B46.5N & -3.674 & 50.9485 & (Smith and Cloutman, 1988) \\
\hline Waun Felen Fignen E13N & -3.674 & 50.9485 & (Smith and Cloutman, 1988) \\
\hline Waun Felen Fignen B90S & -3.674 & 50.9485 & (Smith and Cloutman, 1988) \\
\hline Waun Felen Fignen E188S & -3.674 & 50.9485 & (Smith and Cloutman, 1988) \\
\hline Waun Fignen Felen E188S & -3.674 & 50.9485 & (Smith and Cloutman, 1988) \\
\hline Waun Felen Fignen NE & -3.674 & 50.9485 & (Smith and Cloutman, 1988) \\
\hline Waun Felen Fignen B32S & -3.674 & 50.9485 & (Smith and Cloutman, 1988) \\
\hline Waun Felen Fignen A16W & -3.674 & 50.9485 & (Smith and Cloutman, 1988) \\
\hline Waun Felen Fignen A78E & -3.674 & 50.9485 & (Smith and Cloutman, 1988) \\
\hline Waun Felen Fignen NP & -3.674 & 50.9485 & (Smith and Cloutman, 1988) \\
\hline Waun Felen Fignen E1N & -3.674 & 50.9485 & (Smith and Cloutman, 1988) \\
\hline Waun Felen Fignen SW & -3.674 & 50.9485 & (Smith and Cloutman, 1988) \\
\hline Waun Felen Fignen G00 & -3.674 & 50.9485 & (Smith and Cloutman, 1988) \\
\hline Waun Felen Fignen D7E & -3.674 & 50.9485 & (Smith and Cloutman, 1988) \\
\hline Cefn Glas & -3.5607 & 51.7117 & (Smith and Green, 1995) \\
\hline Cefn Glas & -3.5607 & 51.7117 & (Smith and Green, 1995) \\
\hline Hail Storm Hill & -2.2557 & 53.6663 & (Smith and Taylor, 1989) \\
\hline Low Stony Bank & -2.1268 & 54.0809 & (Smith and Taylor, 1989) \\
\hline Banc Nant Rhys & -3.7284 & 52.3982 & (Smith and Taylor, 1989) \\
\hline Carnedd Wen & -3.5929 & 52.6752 & (Smith and Taylor, 1989) \\
\hline Millteir Cerrig & -3.4572 & 52.8639 & (Smith and Taylor, 1989) \\
\hline Pubble & -7.0891 & 54.9593 & (Smith et al., 1981) \\
\hline Cloghornagh & -7.02 & 54.92 & (Smith et al., 1971b, a) \\
\hline
\end{tabular}

\begin{tabular}{|c|c|c|c|}
\hline $\begin{array}{l}\text { 14C } \\
\text { DATE } \\
\text { (BP) }\end{array}$ & ERROR & $\begin{array}{r}\text { CAL } \\
\text { DATE } \\
\text { (BP) }\end{array}$ & ERROR \\
\hline 2860 & 150 & 2993 & 193 \\
\hline 2630 & 50 & 2748 & 30 \\
\hline 1730 & 50 & 1637 & 66 \\
\hline 880 & 90 & 795 & 92 \\
\hline 3720 & 215 & 4076 & 297 \\
\hline 4935 & & 5667 & 104 \\
\hline 7930 & & 8767 & 170 \\
\hline 4260 & & 4822 & 123 \\
\hline 4270 & & 4834 & 122 \\
\hline 3485 & & 3754 & 131 \\
\hline 7895 & 105 & 8714 & 167 \\
\hline 7250 & & 8064 & 102 \\
\hline 6018 & 90 & 6859 & 117 \\
\hline 7830 & 100 & 8621 & 135 \\
\hline 7020 & 90 & 7850 & 93 \\
\hline 6770 & 90 & 7619 & 69 \\
\hline 6570 & 700 & 7417 & 712 \\
\hline 6520 & 85 & 7429 & 69 \\
\hline 6490 & 90 & 7404 & 77 \\
\hline 6210 & 85 & 7117 & 114 \\
\hline 6210 & 85 & 7117 & 114 \\
\hline 5940 & 90 & 6763 & 108 \\
\hline 5855 & 80 & 6665 & 93 \\
\hline 5790 & 65 & 6590 & 82 \\
\hline 5530 & 85 & 6317 & 74 \\
\hline 5100 & 80 & 5848 & 93 \\
\hline 4900 & 80 & 5631 & 76 \\
\hline 4370 & 80 & 4945 & 122 \\
\hline 1018 & 110 & 930 & 110 \\
\hline 4000 & 70 & 4464 & 94 \\
\hline 4000 & & 4468 & 143 \\
\hline 4500 & & 5156 & 172 \\
\hline 5830 & 50 & 6641 & 59 \\
\hline 1690 & 60 & 1589 & 74 \\
\hline 4170 & 50 & 4732 & 96 \\
\hline 3630 & 50 & 3941 & 69 \\
\hline 2600 & 50 & 2731 & 40 \\
\hline 2900 & 70 & 3034 & 109 \\
\hline 6160 & & 7049 & 136 \\
\hline
\end{tabular}

\begin{tabular}{|c|c|c|c|c|}
\hline TUDE & $\begin{array}{r}\text { Peat } \\
\text { Depth }\end{array}$ & $\begin{array}{l}\text { DATING } \\
\text { METHOD }\end{array}$ & $\begin{array}{l}\text { Age } \\
\text { extra- } \\
\text { polated }\end{array}$ & REGION \\
\hline 173 & 86 & $14 \mathrm{C}$ & $\mathrm{N}$ & N.Scotland \\
\hline 97 & 62 & $14 \mathrm{C}$ & $\mathrm{N}$ & N.Scotland \\
\hline 242 & 33 & $14 \mathrm{C}$ & $\mathrm{N}$ & N.Scotland \\
\hline 169 & 18 & $14 \mathrm{C}$ & $\mathrm{N}$ & N.Scotland \\
\hline \multirow[t]{6}{*}{16} & 325 & $14 \mathrm{C}$ & Y & N.Scotland \\
\hline & & $14 \mathrm{C}$ & & W.Ireland \\
\hline & & $14 \mathrm{C}$ & & W.Ireland \\
\hline & & $14 \mathrm{C}$ & & W.Ireland \\
\hline & & $14 \mathrm{C}$ & & W.Ireland \\
\hline & & $14 \mathrm{C}$ & & W.Ireland \\
\hline 60 & 230 & $14 \mathrm{C}$ & $\mathrm{N}$ & N.Scotland \\
\hline 70 & 105 & POLLEN & $\mathrm{N}$ & N.Scotland \\
\hline 346 & 105 & $14 \mathrm{C}$ & $\mathrm{Y}$ & N.England \\
\hline 480 & 46 & $14 \mathrm{C}$ & $\mathrm{N}$ & Wales \\
\hline 480 & 69 & $14 \mathrm{C}$ & $\mathrm{N}$ & Wales \\
\hline 480 & 90 & $14 \mathrm{C}$ & $\mathrm{N}$ & Wales \\
\hline 480 & 180 & $14 \mathrm{C}$ & $\mathrm{N}$ & Wales \\
\hline 480 & 252 & $14 \mathrm{C}$ & $\mathrm{N}$ & Wales \\
\hline 480 & 19 & $14 \mathrm{C}$ & $\mathrm{N}$ & Wales \\
\hline 480 & 147 & $14 \mathrm{C}$ & $\mathrm{N}$ & Wales \\
\hline 480 & 140 & $14 \mathrm{C}$ & $\mathrm{N}$ & Wales \\
\hline 660 & 74 & $14 \mathrm{C}$ & $\mathrm{N}$ & Wales \\
\hline 480 & 48 & $14 \mathrm{C}$ & $\mathrm{N}$ & Wales \\
\hline 480 & 52 & $14 \mathrm{C}$ & $\mathrm{N}$ & Wales \\
\hline 480 & 76 & $14 \mathrm{C}$ & $\mathrm{N}$ & Wales \\
\hline 500 & 110 & $14 \mathrm{C}$ & $\mathrm{N}$ & Wales \\
\hline 480 & 42 & $14 \mathrm{C}$ & $\mathrm{N}$ & Wales \\
\hline 530 & 47 & $14 \mathrm{C}$ & $\mathrm{N}$ & Wales \\
\hline 480 & 504 & $14 \mathrm{C}$ & $\mathrm{N}$ & Wales \\
\hline 480 & 148 & $14 \mathrm{C}$ & $\mathrm{N}$ & Wales \\
\hline 490 & 147 & $14 \mathrm{C}$ & Y & Wales \\
\hline 491 & 92 & $14 \mathrm{C}$ & Y & Wales \\
\hline 460 & 155 & $14 \mathrm{C}$ & $\mathrm{N}$ & N.England \\
\hline 400 & 88 & $14 \mathrm{C}$ & $\mathrm{N}$ & N.England \\
\hline 543 & 185 & $14 \mathrm{C}$ & $\mathrm{N}$ & Wales \\
\hline 520 & 100 & $14 \mathrm{C}$ & $\mathrm{N}$ & Wales \\
\hline 490 & 100 & $14 \mathrm{C}$ & $\mathrm{N}$ & Wales \\
\hline 213 & 50 & $14 \mathrm{C}$ & $\mathrm{N}$ & N.Ireland \\
\hline & & $14 \mathrm{C}$ & & W.Ireland \\
\hline
\end{tabular}




\begin{tabular}{|c|c|c|c|}
\hline SITE NAME & LONGITUDE & LATITUDE & REFERENCE \\
\hline Crockbrack & -6.88 & 54.8 & (Smith et al., 1971b, a) \\
\hline Leaghs Bridge & -6.92 & 54.78 & (Smith et al., 1971b, a) \\
\hline Salvin Ridge SR3 & -1.71 & 53.4406 & (Tallis, 1985) \\
\hline Featherbed Top FT1 & -1.71 & 53.4406 & (Tallis, 1985) \\
\hline Robinson's Moss AT1 & -1.8359 & 53.4157 & (Tallis, 1991) \\
\hline Featherbed Moss AT12 & -1.71 & 53.4406 & (Tallis, 1991) \\
\hline Alport Moor AM & -1.9336 & 53.4976 & (Tallis, 1991) \\
\hline Robinsons Moss AT4 & -1.9336 & 53.4976 & (Tallis, 1991) \\
\hline Robinson's Moss AT5 & -1.9336 & 53.4976 & (Tallis, 1991) \\
\hline Featherbed Moss FW3 & -1.71 & 53.4406 & (Tallis, 1991) \\
\hline Featherbed Moss FW4 & -1.71 & 53.4406 & (Tallis, 1991) \\
\hline Tintwistle High Moor AT7 & -1.9478 & 53.49 & (Tallis, 1991) \\
\hline Tintwistle High Moor AT8 & -1.9478 & 53.49 & (Tallis, 1991) \\
\hline Tintwistle High Moor AT9 & -1.9478 & 53.49 & (Tallis, 1991) \\
\hline Arnfield AT11 & -1.95 & 53.4872 & (Tallis, 1991) \\
\hline Robinson's Moss AT6 & -1.9336 & 53.4976 & (Tallis, 1991) \\
\hline Featherbed Moss AT13 & -1.71 & 53.4406 & (Tallis, 1991) \\
\hline Lady Clough Moor & -1.8434 & 53.4292 & (Tallis and Switsur, 1983) \\
\hline Laund Clough & -1.7633 & 53.4884 & (Tallis and Switsur, 1983) \\
\hline Coldharbour Moor & -1.8916 & 53.4319 & (Tallis and Switsur, 1983) \\
\hline Featherbed Moss (East) & -1.854 & 53.4319 & (Tallis and Switsur, 1983) \\
\hline Robinson's Moss & -1.9336 & 53.4976 & (Tallis and Switsur, 1990) \\
\hline Tintwistle Knarr & -1.9336 & 53.4976 & (Tallis and Switsur, 1990) \\
\hline Clarach & -4.0514 & 52.4847 & (Taylor, 1973) \\
\hline Glaslyn & -4.0667 & 53.0708 & (Taylor, 1975) \\
\hline Carneddau 1 & -3.492 & 52.5794 & (Walker, 1993) \\
\hline Carneddau 2 & -3.492 & 52.5794 & (Walker, 1993) \\
\hline Carneddau 4 & -3.492 & 52.5794 & (Walker, 1993) \\
\hline Carneddau 3 & -3.492 & 52.5794 & (Walker, 1993) \\
\hline Carneddau 5 & -3.492 & 52.5794 & (Walker, 1993) \\
\hline Beaghs Forest & -6.18 & 55.07 & (Zurek, 1984) \\
\hline Beaghs Sandpit & -6.18 & 55.08 & (Zurek, 1984) \\
\hline Breen & -6.23 & 55.15 & (Zurek, 1984) \\
\hline Crocknamoyle & -6.15 & 55.12 & (Zurek, 1984) \\
\hline Gruig Top & -6.13 & 55.1 & (Zurek, 1984) \\
\hline Loughaveema & -6.12 & 55.15 & (Zurek, 1984) \\
\hline
\end{tabular}

\begin{tabular}{|c|c|c|c|}
\hline $\begin{array}{l}\text { 14C } \\
\text { DATE } \\
\text { (BP) }\end{array}$ & ERROR & $\begin{array}{r}\text { CAL } \\
\text { DATE } \\
\text { (BP) }\end{array}$ & ERROR \\
\hline 6250 & & 7164 & 123 \\
\hline 7640 & & 8433 & 83 \\
\hline 6300 & & 7221 & 113 \\
\hline 5500 & & 6291 & 92 \\
\hline 9100 & & 10255 & 101 \\
\hline 8200 & & 9158 & 144 \\
\hline 8100 & & 9024 & 139 \\
\hline 7800 & & 8585 & 122 \\
\hline 7600 & & 8401 & 81 \\
\hline 6800 & & 7643 & 81 \\
\hline 6200 & & 7101 & 132 \\
\hline 6000 & & 6838 & 127 \\
\hline 5500 & & 6291 & 92 \\
\hline 5400 & & 6194 & 114 \\
\hline 5400 & & 6194 & 114 \\
\hline 5400 & & 6194 & 114 \\
\hline 5400 & & 6194 & 114 \\
\hline 5410 & 50 & 6221 & 55 \\
\hline 5110 & 50 & 5866 & 62 \\
\hline 4670 & 40 & 5396 & 62 \\
\hline 4570 & 65 & 5275 & 108 \\
\hline 8950 & 80 & 10115 & 125 \\
\hline 4475 & 55 & 5119 & 123 \\
\hline 5970 & 90 & 6799 & 112 \\
\hline 4220 & 100 & 4776 & 131 \\
\hline 9200 & & 10361 & 129 \\
\hline 9200 & & 10361 & 129 \\
\hline 7000 & & 7829 & 103 \\
\hline 4710 & 80 & 5445 & 103 \\
\hline 3450 & 70 & 3707 & 93 \\
\hline 2620 & & 2731 & 105 \\
\hline 4040 & & 4524 & 149 \\
\hline 2890 & & 3024 & 145 \\
\hline 2930 & & 3082 & 149 \\
\hline 3555 & & 3845 & 133 \\
\hline 2885 & & 3017 & 144 \\
\hline
\end{tabular}

\begin{tabular}{|c|c|c|c|c|}
\hline UDE & $\begin{array}{r}\text { Peat } \\
\text { Depth }\end{array}$ & $\begin{array}{l}\text { DATING } \\
\text { METHOD }\end{array}$ & $\begin{array}{l}\text { Age } \\
\text { extra- } \\
\text { polated }\end{array}$ & REGION \\
\hline & & $14 \mathrm{C}$ & & W.Ireland \\
\hline & & $14 \mathrm{C}$ & & W.Ireland \\
\hline 525 & 220 & POLLEN & $\mathrm{N}$ & N.England \\
\hline 540 & 150 & POLLEN & $\mathrm{N}$ & N.England \\
\hline 485 & 450 & POLLEN & Y & N.England \\
\hline 540 & & POLLEN & $\mathrm{Y}$ & N.England \\
\hline 540 & 320 & POLLEN & & N.England \\
\hline 495 & 335 & POLLEN & & N.England \\
\hline 485 & 240 & POLLEN & $\mathrm{Y}$ & N.England \\
\hline 510 & 270 & POLLEN & & N.England \\
\hline 485 & 250 & POLLEN & & N.England \\
\hline 467 & 257 & POLLEN & & N.England \\
\hline 465 & 230 & POLLEN & & N.England \\
\hline 435 & 150 & POLLEN & & N.England \\
\hline 450 & & POLLEN & $\mathrm{Y}$ & N.England \\
\hline 485 & 180 & POLLEN & Y & N.England \\
\hline 540 & & POLLEN & Y & N.England \\
\hline 480 & 120 & $14 \mathrm{C}$ & $\mathrm{N}$ & N.England \\
\hline 455 & 150 & $14 \mathrm{C}$ & $\mathrm{N}$ & N.England \\
\hline 435 & 90 & $14 \mathrm{C}$ & $\mathrm{N}$ & N.England \\
\hline 470 & 50 & $14 \mathrm{C}$ & $\mathrm{N}$ & N.England \\
\hline 500 & 410 & $14 \mathrm{C}$ & $\mathrm{N}$ & N.England \\
\hline \multirow[t]{2}{*}{500} & 70 & $14 \mathrm{C}$ & $\mathrm{N}$ & N.England \\
\hline & & $14 \mathrm{C}$ & $\mathrm{N}$ & Wales \\
\hline 480 & 120 & $14 \mathrm{C}$ & $\mathrm{N}$ & Wales \\
\hline 400 & 220 & POLLEN & $\mathrm{N}$ & Wales \\
\hline 400 & 125 & POLLEN & $\mathrm{N}$ & Wales \\
\hline 400 & 155 & POLLEN & $\mathrm{N}$ & Wales \\
\hline 400 & 115 & $14 \mathrm{C}$ & $\mathrm{N}$ & Wales \\
\hline \multirow[t]{7}{*}{400} & 65 & $14 \mathrm{C}$ & $\mathrm{N}$ & Wales \\
\hline & & $14 \mathrm{C}$ & & W.Ireland \\
\hline & & $14 \mathrm{C}$ & & W.Ireland \\
\hline & & $14 \mathrm{C}$ & & W.Ireland \\
\hline & & $14 \mathrm{C}$ & & W.Ireland \\
\hline & & $14 \mathrm{C}$ & & W.Ireland \\
\hline & & $14 \mathrm{C}$ & & W.Ireland \\
\hline
\end{tabular}




\section{References:}

Ashmore, P., Brayshay, B. A., Edwards, K. J., Gilbertson, D. D., Grattan, J. P., Kent, M., Pratt, K. E., and Weaver, R. E.: Allochthonous and autochthonous mire deposits, slope instability and palaeoenvironmental investigations in the Borve Valley, Barra, Outer Hebrides, Scotland, The Holocene, 10, 97-108, 10.1191/095968300676239809, 2000.

Barber, J. W., and Brown, M. M.: An Sithean, Islay, Proc Soc Antiq Scot, 114, 161-188, 1984.

Bartley, D. D., and Chambers, C.: A pollen diagram, radiocarbon ages and evidence of agriculture on Extwistle Moor, Lancashire, New Phytologist, 121, 311-320, 10.1111/j.1469-8137.1992.tb01117.x, 1992.

Birks, H. H.: Studies in the vegetational history of Scotland IV: Pine stumps in Scottish blanket peats, Philosophical Transactions of the Royal Society of London. Series B, Biological Sciences, B 270, 181-226, 1975.

Blackford, J. J.: Blanket mires and climatic change: a palaeoecological study based on peat humification and microfossil analysis, PhD, Keele University, UK, 1990.

Bohncke, S. J. P.: Vegetation and habitation history of the Callanish area, Isle of Lewis, Scotland, in: The Cultural Landscape - Past, Present and Future, edited by: Birks, H. H., Birks, H. J. B., Kaland, P. E., and Moe, D., Cambridge University Press, Cambridge, 445-461, 1988.

Bostock, J. L.: The history of the vegetation of the Berwyn Mountains, North Wales, with emphasis on the development of the blanket mire, PhD, University of Manchester, 1980.

Bridge, M. C., Haggart, B. A., and Lowe, J. J.: The History and Palaeoclimatic Significance of Subfossil Remains of Pinus Sylvestris in Blanket Peats From Scotland, Journal of Ecology, 78, 77-99, 10.2307/2261038, 1990.

Caseldine, C., Thompson, G., Langdon, C., and Hendon, D.: Evidence for an extreme climatic event on Achill Island, Co. Mayo, Ireland around 5200-5100 cal. yr BP, Journal of Quaternary Science, 20, 169-178, 10.1002/jqs.901, 2005.

Chambers, F. M.: Date of blanket peat initiation in upland South Wales, Quaternary Newsletter, 35, 24-29, 1981.

Chambers, F. M.: Two radiocarbon-dated pollen diagrams from high-altitude blanket peats in South Wales, The Journal of Ecology, 445-459, 1982.

Chambers, F. M.: Three Radiocarbon-Dated Pollen Diagrams from Upland Peats North-West of Merthyr Tydfil, South Wales, Journal of Ecology, $71,475-487,10.2307 / 2259729,1983$.

Chambers, F. M., Barber, K. E., Maddy, D., and Brew, J.: A 5500-year proxy-climate and vegetation record from blanket mire at Talla Moss, Borders, Scotland, Holocene, 7, 391-399, 10.1177/095968369700700402, 1997. 
Chapman, S. B.: The Ecology of Coom Rigg Moss, Northumberland: II. The Chemistry of Peat Profiles and the Development of the Bog System, Journal of Ecology, 52, 315-321, 10.2307/2257598, 1964.

Charman, D. J.: Origins and development of the Flow Country blanket mire, Northern Scotland, with particular reference to patterned fens, PhD, University of Southampton, 1990.

Charman, D. J.: Blanket mire formation at the Cross Lochs, Sutherland, northern Scotland, Boreas, 21, 53-72, 10.1111/j.15023885.1992.tb00013.x, 1992

Charman, D. J.: Late-glacial and Holocene vegetation history of the Flow Country, northern Scotland, New Phytologist, 127, 155-168, 1994.

Cundill, P. R.: The distribution, age and formation of blanket peat on the North York Moors, Proceedings of the North of England Soils Discussion Group 9, 25-29, 1977.

Dresser, Q.: University College Cardiff Radiocarbon dates I, Radiocarbon, 27, 338-385, 1985.

Dubois, A. D., and Ferguson, D. K.: The climatic history of pine in the cairngorms based on radiocarbon dates and stable isotope analysis, with an account of the events leading up to its colonization, Review of Palaeobotany and Palynology, 46, 55-80, http://dx.doi.org/10.1016/00346667(85)90038-7, 1985.

Dwyer, R. B., and Mitchell, F. J. G.: Investigation of the environmental impact of remote volcanic activity on north Mayo, Ireland, during the midHolocene, The Holocene, 7, 113-118, 10.1177/095968369700700111, 1997.

Edmonds, M., Sheridan, A., and Tipping, R.: Survey and excavation at Creag na Caillich, Killin, Perthshire, Proc Soc Antiq Scot , 122, 77-112, 1992 .

Edwards, K. J., Hirons, K. R., and Newell, P. J.: The palaeoecological and prehistoric context of minerogenic layers in blanket peat: a study from Loch Dee, southwest Scotland, The Holocene, 1, 29-39, 10.1177/095968369100100105, 1991.

Ellis, C. J., and Tallis, J. H.: Climatic control of peat erosion in a North Wales blanket mire, New Phytologist, 152, 313-324, 10.1046/j.0028646X.2001.00259.x, 2001.

Ellis, C. J.: Interactions between hydrology, burning and contrasting plant groups during the millennial-scale development of sub-montane wet heath, Journal of Vegetation Science, 19, 693-U657, 10.3170/2008-8-18439, 2008.

Eydt, H. R.: A pollen diagram from a blanket bog in the Campsie Fells Trans. bot. Soc. Edinb., 39, 1, 1960.

Fyfe, R. M., and Woodbridge, J.: Differences in time and space in vegetation patterning: analysis of pollen data from Dartmoor, UK, Landscape Ecol, 27, 745-760, 10.1007/s10980-012-9726-3, 2012. 
Goddard, A.: Studies of the vegetational changes associated with the initiation of blanket peat accumulation in North-East Ireland, PhD, Queen's University, Belfast, 1971.

Godwin, H., and Willis, E. H.: Studies of the post-glacial history of British vegetation. III. Fenland pollen diagrams. IV. Post glacial changes of relative land and sea-level in the English fenland, Philosophical Transactions of the Royal Society of London. Series B, Biological Sciences, B.230, 239-303, 1961.

Hakansson, S.: University of Lund Radiocarbon Dates XVIII, Radiocarbon, 28, 141-164, 1986.

Harkness, D. D.: Scottish Universities Research and Reactor Centre, radiocarbon measurements IV, Radiocarbon, 23, 252-304, 1981.

Honeyman, A.: Studies in the Holocene vegetation history of Wensleydale, School of Geography, University of Leeds, Leeds, 323 pp., 1985.

Jones, V. J.: A palaeoecological study of the post-glacial acidification of the Round Loch of Glenhead and its catchment, PhD, University of London, 1987.

Keatinge, T. H., and Dickson, J. H.: Mid-Flandrian changes in vegetation on mainland Orkney, New Phytologist, 82, 585-612, 10.2307/2433557, 1979.

Langdon, P. G., and Barber, K. E.: New Holocene tephras and a proxy climate record from a blanket mire in northern Skye, Scotland, Journal of Quaternary Science, 16, 753-759, 10.1002/jqs.655, 2001.

Maguire, D. J.: The inception and growth of blanket peat: a study of northern Dartmoor, PhD, University of Bristol, 1983.

Mighall, T. M., and Chambers, F. M.: The environmental impact of prehistoric copper mining at Copa Hill, Cwmystwyth, Wales, The Holocene, 3, 260-264, 1993.

Mills, C.: Unpublished data.

Moar, N. T.: A radiocarbon-dated pollen diagram from North-West Scotland, New Phytologist, 68, 209-214, 10.1111/j.1469-8137.1969.tb06434.x, 1969.

O'Connell, M.: Origins of lowland Irish blanket bog, in: Ecology and Conservation of Irish Peatlands, edited by: Doyle, G. J., Royal Irish Academy, Dublin, 49-71, 1990.

Robinson, D.: Investigations into the Aukhorn peat mounts, Keiss, Caithness: pollen, plant macrofossil and charoal analyses, New Phytologist, 106, 185-200, 10.1111/j.1469-8137.1987.tb04802.x, 1987.

Simmons, I. G., and Innes, J. B.: Tree remains in a North York Moors peat profile, Nature, 294, 76-78, 1981. 
Smith, A. G., Pearson, G. W., and Pilcher, J. R.: BELFAST RADIOCARBON DATES IV, Radiocarbon, 13, 450-\&, 1971a.

Smith, A. G., Pearson, G. W., and Pilcher, J. R.: BELFAST RADIOCARBON DATES III, Radiocarbon, 13, 103-\&, 1971b.

Smith, A. G., Gaskell-Brown, C., Goddard, I. C., Goddard, A., Pearson, G. W., and Dresser, P. Q.: Archaeology and environmental history of a barrow at Pubble, Loughermore, Townland, County Londonderry., Proceedings of the Royal Irish Academy, 81C, $29-66,1981$.

Smith, A. G., and Cloutman, E. W.: Reconstruction of Holocene vegetation history in three dimensions at Waun-Fignen-Felen, an upland site in South Wales, Philosophical Transactions of the Royal Society of London. Series B, Biological Sciences, 322, 159-219, 10.2307/2398747, 1988.

Smith, A. G., and Green, C. A.: Topogenous peat development and late- Flandrian vegetation history at a site in upland South Wales, The Holocene, 5, 172-183, 10.1177/095968369500500205, 1995.

Smith, R. T., and Taylor, J.: Biopedological processes in the inception of peat formation, International Peat Journal, 3, 1-24, 1989.

Tallis, J. H., and Switsur, V. R.: Forest and Moorland in the South Pennine Uplands in the Mid-Flandrian Period: I. Macrofossil Evidence of the Former Forest Cover, Journal of Ecology, 71, 585-600, 10.2307/2259736, 1983.

Tallis, J. H.: Mass Movement and Erosion of a Southern Pennine Blanket Peat, Journal of Ecology, 73, 283-315, 10.2307/2259784, 1985.

Tallis, J. H., and Switsur, V. R.: Forest and Moorland in the South Pennine Uplands in the Mid-Flandrian Period.: II. The Hillslope Forests, Journal of Ecology, 78, 857-883, 10.2307/2260940, 1990.

Tallis, J. H.: Forest and moorland in the South Pennine uplands in the Mid-Flandrian Period .3. The spread of moorland local regional and national, Journal of Ecology, 79, 401-415, Doi 10.2307/2260722, 1991.

Taylor, J.: Chronometers and chronicles: a study of palaeo-environments in west Central Wales, Progress in Geography, 5, 247-334, 1973.

Taylor, J.: The distribution of peat deposits in the British Isles, in: Soil organic matter; ed. by R.G. Wyn Jones, 2-15; Welsh Soils Discussion Group, Report No. 16, 1975.

Walker, M. J. C.: Holocene (Flandrian) vegetation change and human activity in the Carneddau area of upland mid-Wales, in: Climate Change and Human Impact on the Landscape, edited by: Chambers, F. M., Springer Netherlands, 169-183, 1993.

Zurek, S.: Organic matter accumulation in European peatlands (on the basis of 14C data), 7th International Peat Congress, Dublin, 1984. 